\title{
The airway epithelium dysfunction in the pathogenesis of asthma: The evidence
}

\author{
Salvatore Leonardi ${ }^{1^{*}}$, Alfina Coco ${ }^{1}$, Michele Miraglia Del Giudice ${ }^{2}$, Gianluigi L. Marseglia ${ }^{3}$, \\ Mario La Rosa ${ }^{1}$ \\ ${ }^{1}$ Pediatric Bronchopneumology and Cystic Fibrosis Unit, Department of Medical and Paediatric Sciences, University of Catania, \\ Catania, Italy; ${ }^{*}$ Corresponding Author: leonardi@unict.it \\ ${ }^{2}$ Clinical Pediatric, 2nd University of Naples, Naples, Italy \\ ${ }^{3}$ IRCCS University Hospital, San Martino, University of Pavia, Pavia, Italy
}

Received 3 December 2012; revised 2 January 2013; accepted 9 January 2013

\section{ABSTRACT}

Asthma is traditionally defined as a chronic, multisystem, multicellular disease characterized by bronchial hyper-responsiveness (BHR) and lung inflammation. In this illness is particularly involving the airway epithelium as the place where the inflammation begins, develops and often returns through airway remodelling. In specific way there is also an evolving awareness of the active participation of structural elements, such as the airway epithelium, airway smooth muscle, and endothelium, in this process. The airway inflammation and remodelling together likely explain the clinical manifestations of asthma that is demonstrated by complicated respiratory manifestations in which wheeze occurring secondary to bronchoconstriction in the setting of airway hyper-responsiveness and mucous hypersecretion. This is confirmed by also the histopathological analyses of bronchial biopsies from asthmatic subjects. For this reason is very important to understand the physiological role of the airway epithelium and their changes during the exacerbation of disease.

Keywords: Airway Epithelium; Dysfunction; Asthma; Evidence

\section{INTRODUCTION}

Asthma is traditionally defined as a chronic, multisystem, multicellular disease characterized by bronchial hyper-responsiveness (BHR) and lung inflammation particularly within the airways [1]. The development of asthma requires an interaction between the environment and genetic susceptibility in which there is a sharing between the innate and acquired immune system in some of the clinical manifestations of the disease, particularly in the development of severe exacerbations. The clinical manifestations of asthma characterised by repeated, variable, episodic attacks of dyspnea, cough and bronchoconstriction of airway are expression of inflammatory cell infiltration together airway remodelling. In specific way, in addition to respiratory involvement in which there is participation of the airway epithelium, airway smooth muscle, and endothelium, in both allergic and non-allergic forms of the disease, the airway is infiltrated by T-helper (Th) cells. These cells predominantly secrete characteristic cytokines such as interleukin (IL)-4, IL-5, and IL-13, the so-called Th2 cytokine milieu. These cytokines stimulate mast cells, cause eosinophilia, promote leukocytosis, and enhance B-cell IgE production, and may also participate in the characteristic airway remodelling of asthma. However, for an individual to develop an asthmatic phenotype is required the combination of both exposure to appropriate stimuli and a genetic predisposition [2,3]. It's important to underline is the role of airway smooth muscle (ASM) that in airway remodelling phase maybe induce to airflow obstruction observed in severe form of asthma [4-7]. In fact it's probable that the recurrence the ASM remodelling process is linked with relationship between ASM cells and basal steam cells [8]. These details are essential to explain the significance of the review aimed and to understand the role of epithetlium in the airways. Understanding of role of the epithetlium there is also used to find out all new methods like specific immunotherapy (SIT), in particular sublingualswallow immunotherapy (SLIT) to overcome the problem of the evolution of asthma.

\section{AIRWAY CELLS PHENOTYPE IN ASTHMATIC EPITHELIUM}

Airway epithelium forms a continuous, highly regulated physical barrier lining of the airway lumen, which prevents invasion of inhaled environmental agents such as aeroallergens, pollutants and pathogens. It's probably 
that the loss of mucosal barrier function in asthma might result from increased fragility of the airway epithelium and/or an impaired repair mechanism with inability to restore cell-cell contacts upon damage [9]. The way in which the airway epithelium is disrupted in asthma is must be include the detachment of columnar ciliated cells, increased permeability to allergens, and alteration expression of the junction molecule E-cadherin at sites of epithelial detachment. Is demonstrated that the expression of the junction molecules E-cadherin and zonaoccludens-1 (ZO-1) is reduced in vitro in differentiated bronchial epithelium from asthma patients compared with healthy people, which indicates that asthma epithetlium inefficiently forms intercellular contacts [9-14]. By contrast, increased expression of repair mediators, including epidermal growth factor receptor (EGFR) and transforming growth factor (TGF) $\beta$ at sites of ciliated cell detachment supports a role for aberrant repair in the asthmatic epithelial phenotype [15]. This was confirmed as asthmatic airway epithelium has been demonstrated to contain higher numbers of cells that express the basal cell markers cytokeratin-5 and-14 and p63 [9], which represent the main transient amplifying cell population in the airway epithelium. These data imply that aberrant epithelial repair processes in asthma contribute to epithelial damage and loss of effective barrier function. Further different theories, many studious have identified asthma susceptibility genes including DPP10, GPCRA, CHI3L1 and a proto-cadherin gene family member PCDH1 are expressed in airway-epithelial cells [16]. Moreover, biopsy studies in children suggest that the damaged epithelial phenotype occurs early in disease pathogenesis, even before a definitive diagnosis of asthma [17]. Multiple asthma biopsy studies have demonstrated airway epithetlial abnormality, and the epithelium, placed at the interface between the external environment and the host, appears to be both a site of action and of reaction within the asthmatic inflammatory cascade [17-23] (Table 1). In specific way is discovered that epithelial cells possess microbial detection mechanisms, and they express a tightly regulated and specifically localized set of pattern recognition receptors (PRRs) as well as direct antimicrobial effector. This cell-type-specific innate immune recognition equipment enables them to respond to antigens and allergens, thereby initiating the first step in the hostpathogen interaction. Is underline that the barrier function of the epithelia material and tissue homeostasis depends on the integrity of the cells and the expression and interaction of the proteins, cell against cell, on junctonal complexes and in specific way on tight junctions (TIs), that moreover induce the susceptibility to environmental agents [24]. Current observations point to the idea that the epithelial barrier influences besides the development of antigen-specific immunity, suggesting that the type of immune response elicited by the host highly depends on the nature of the response defined by the local tissue microenvironment. It is therefore very likely that innate immune recognition by barrier cells determines the functional properties of tissue residing DCs, thereby instructing the outcome of antigen specific immunity [25]. This concept has important implications for the regulation of mucosal homeostasis and for the initiation of innate and adaptive immune responses. The airway epithelium is known to be a major source of pro-inflammatory mediators. Recent examples include thymic stromal lymphopoietin, an epithelial derived cytokine expressed in the asthmatic airway, which has been shown to activate dendritic cells, promote Th2 responses, and activate mast cells [26-28]. Endothelin-1 is also increased in airway epithelial biopsies in patients with severe asthma and in particular in patients with steroid-resistant asthma [29]. Particulate matter has also been shown to induce the release of pro-inflammatory mediators and induce cycloxygenase-2 expression in human airway epithelial cells [30]. In addition to acting as a potent source of pro-inflammatory cytokines, epithelial cells possess the ability to present self-antigen, with resultant effects on the regulation of CD4+ T-cell function, including the induction of Foxp3 Treg cells, thus promoting immune tolerance [31]. In fact the process of airway remodelling involves alterations to the various components of airway structure, with fibroproliferation, influx of myofibroblasts, collagen deposition, release inflammatory mediators, hypertrophy of airway smooth muscle, and reticular basement membrane thickening typical (Figure 1) In particularly, is demonstrated that in asthmatic airway, there are increased numbers of sub epithelial myofibroblasts in the airway mucosa, an increase of proliferation and thickening of epithelium like also lamina reticularis and an fibroblastic infiltration of the lung that conduce to severe disease [9,23,32]. While studies demonstrate a progressive loss of lung function associated with severe asthma exacerbations it has been recognized that remodelling may occur very early in asthma and may in some cases even predate clinical symptoms like confirm airway biopsy in children in which pathologic changes occur early in the asthmatic airway [33-35] (Figure 2). The function of the impaired lung is due to fibroblastic infiltration may plausibly be secondary to the recruitment of circulating bone marrow-derived progenitors termed fibrocytes to the airway and to the proliferation and expansion of resident fibroblasts, or possibly, epithelial cells may undergo phenotypic change to effector fibroblasts through a process termed epithelial-mesenchymal transition. Moreover airway biopsies have revealed the increased presence of fibrocytes in the airway smooth muscle bundle of patients with asthma of varying severity compared with control subjects [36]. Airway smooth muscle cells have many roles and can also be induced to secrete mediators that may promote mast cell chemotaxis, proliferation, 
Table 1. Airway epithelium modifications and severe airway diseases.

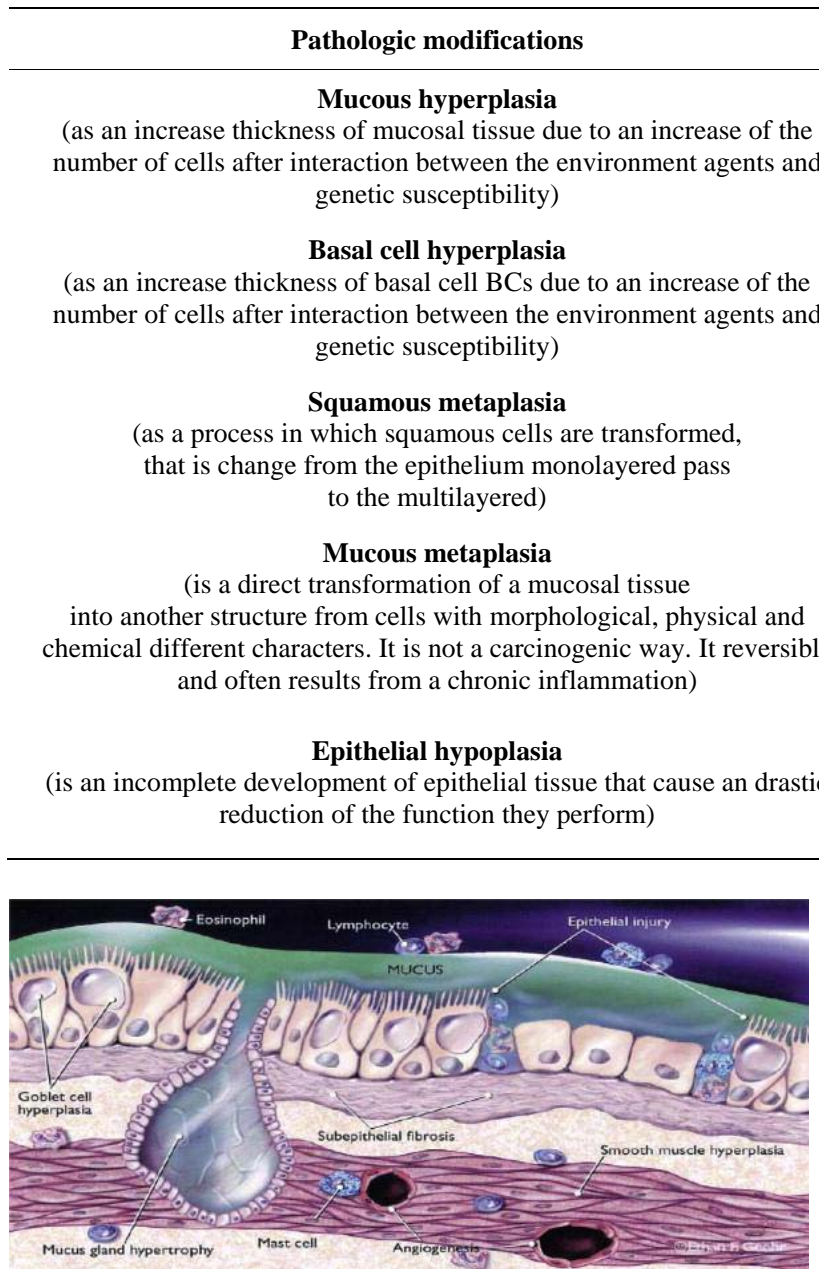

Figure 1. Graphic representation of airway cells phenotype in asthmatic epithelium. This figure show the alterations to the various components of airway structure, with fibroproliferation, influx of myofibroblasts, collagen deposition, globet cell hyperplasia, release inflammatory mediators, hypertrophy of airway smooth muscle and reticular basement membrane.

survival and activated complement-induced mast cell degranulation $[37,38]$. Bronchial epithelial cell experiments have demonstrated a role for TLR signalling in the activation of epidermal growth factor receptor, suggesting a role for TLRs in potentiating remodelling. [39]. Histamine is capable of inducing the transition from fibroblasts to myofibroblasts, as measured by a smooth muscle actin expression, and can, in addition, induce connective-tissue-growth-factor expression in fibroblasts, suggesting the ability to participate in the process of remodelling [40]. Airway smooth muscle mass is increased in the asthmatic airway. These smooth muscle cells shows increased expression of the fast myosin heavy chain isoform transgelin, as well as myosin light chain kinase, with further experiments in rat models suggesting that this may be associated with the potential to increase
Disorders in which change has been reported

Asthma, COPD, cystic fibrosis (larger airways)

COPD, cystic fibrosis

COPD, Cystic Fibrosis

Asthma, COPD

Bronchiolitis obliterans

Severe form of bronchiolitis that may occur with extensive lesions of bronchiolar mucosa, obliteration of the lumen and fibrous reaction that can lead to severe respiratory failure

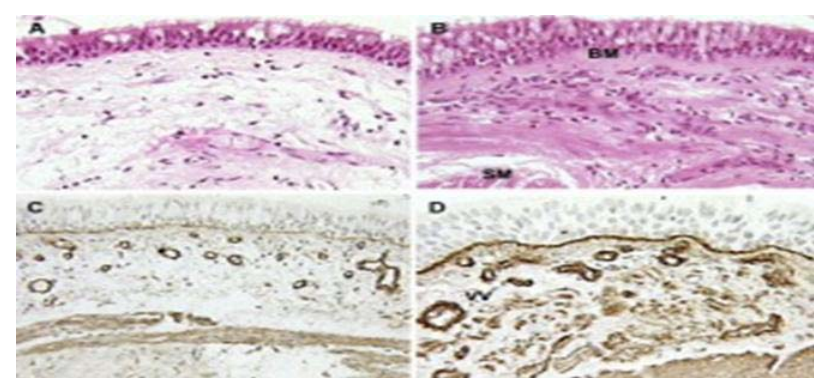

Figure 2. Sections of normal bronchial wall and that from an asthmatic with airway remodelling changes. In this figure obtained by histological exam: (A) Stained airway from normal control; (B) Stained airway in severe asthma. Note thickened subepithelial collagen below the basement membrane (BM), inflammatory cells between the smooth muscle layer (SM) and goblet cell hyperplasia in epithelium; (C) Collagen IV staining of a control airway to demonstrate basement membrane; (D) Collagen IV staining of asthmatic airway showing true basement membrane beneath epithelium and vessels (VV) in submucosa.

maximal flow and thereby contribute to the airway hyper-responsiveness seen in asthma [41]. The other important characterized is that can promote in vitro angiogenesis [42], demonstrated thanks to BAL fluid obtained from patients with asthma has been shown to possess a pro-angiogenic effect, which appears to be mediated through the actions of vascular endothelial growth factor (VEGF), while further studies of the asthmatic airway demonstrate increased vascularity and higher levels of VEGF, and potentially implicate mast cells as a signifycant source of VEGF [42,43]. However, increased vascularity and higher VEGF levels exist both in patients with asthma and patients with eosinophilic bronchitis but 
without asthma, implying that vascular remodelling may not directly influence airway hyper-responsiveness. Studies point to an interactive process between the immunologic and neuronal systems in the propagation of asthmatic response. Murine models of asthma have shown that the communicative process between the two systems may be linked via the ion channel TRPA1. In human studies, increased levels of brain derived neurotrophic factor have been associated with loss of control in patients with mild allergic asthma [44].

\section{AIRWAY BASAL STEAM CELLS IN EPITHELIAL HOMEOSTASIS AND REMODELLING}

Basal cells (BCs) are a common feature of stratified and pseudostratified epithelia throughout the body (Figure 3). The cytoskeletal, junctional and adhesive proteins of BCs help to anchor the epithelium to the matrix and insulate the underlying stroma from the external environment. These cells are a population of multipotent stem cells that drives both homeostasis of the normal epithelium and probably contribute to disease susceptibility, initiation and progression. It's for this reason that the disruption of the normal balance between BC proliferation and differentiation can lead, at two extremes, to BC hyperplasia or epithelial hypoplasia. It's probable because the alterations in Bcs' genomes through mutations or epigenetic modifications induced by environ-

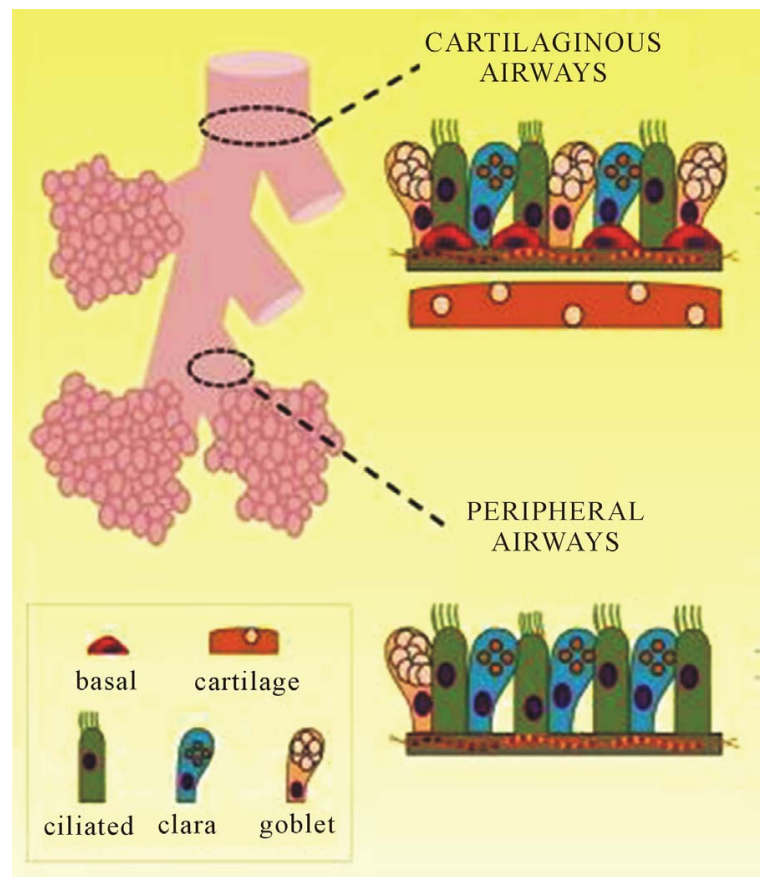

Figure 3. Imagine of section of airway including basal steam cells. This figure show the basal cells (BCs) of airway that are a common feature of stratified and pseudostratified epithelia throughout the body during involve of disease. mental agents might affect the long-term susceptibility of individuals to respiratory disease. For this reason changes in BCs or their progeny could theoretically lead to pathological airway remodelling in a number of different ways. Such changes in the behavior of BCs and/or their putative multipotent progeny might result from intrinsic (including epigenetic) alterations in their transcriptional and regulatory programs, which in turn affect proliferative and differentiation potential. Alternatively, or in addition, the changes might result from altered paracrine signaling within the dynamic and complex BC niche. In every way, it's probable that epithelial changes and/or alter epithelial-mesenchymal interactions further contribute to disease by exacerbating pathological changes in the mesenchyme, including inflammation, fibrosis and smooth muscle hyperplasia. The data suggest that hyperproliferating BCs secrete cytokines, such as interleukin$1 \beta$ (IL-1 $\beta$ ), that promote airway wall fibrosis [45]. Likewise, the generation of stratified suprabasal cells at the expense of luminal cells might lead to squamous metaplasia, whereas a failure to proliferate, increased apoptosis or inappropriate commitment to differentiation at the expense of self-renewal could all contribute to epithelial hypoplasia. These changes can, in turn, disrupt the reciprocal trophic interactions between the epithelium and mesenchyme, and exacerbate submucosal remodeling [46]. Thus, a greater understanding of BC behavior is potentially of clinical relevance. For example, therapies aimed at regulating BC proliferation and directing their differentiation towards specific lineages might help to restore a normal phenotype in a disease context, and as they are a long-lived population, gene or cellular replacement therapies targeting them are likely to provide sustained rather than transient remediation [47]. In conclusion there is asthma without impairment of the barrier function. In fact, the knowledge of the pathological role of the airway epithelium in developing asthma could be useful to physicians to develop target drugs in order to better treatment and the management of bronchial hyper-reactivity.

\section{CONCLUSIONS}

Asthma is a complex inflammatory disorder that if left untreated asthma tends to recur because the remodeling process is continuous. This is related to the important interactions between the innate and acquired immune system in some clinical manifestations of disease, particularly in the development of severe exacerbations. For this reason American studies have deepened the role of specific immunotherapy (SIT) to overcome the problem of the evolution of asthma. Oral mucosal tissue displays high permeability for allergens. In particular the presence in oral mucosa of dendritic cell (DCs) which express the high-affinity receptor for immunoglobulin Ig-E (FceRI) 
seems to suggest that the generation of $\mathrm{T}$ regulatory cells in periphery is orchestrated by a particular subset of DCs. These structural and functional abnormalities might lead to enhanced signalling between the epithelium and underlying immune and structural cells. In these regard studies about specific immunotherapy (SIT) has been shown to be safe and effective in the treatment of clinically significant respiratory IgE-mediated diseases and it represents the only approach currently available to redirect inappropriate immune response in atopic patients. For this reason sublingual-swallow immunotherapy (SLIT) considered like an alternative way to subcutaneous immunotherapy, has been shown to be safe and effective in several double blind placebo-controlled studies in children affected by asthma and rhinitis and in 2001 the guidelines on allergic rhinitis and its impact on asthma (ARIA) extended the indications of SLIT also to children [48-53]. The SLIT, born in the 80s of the last century is now widely used with the use of high doses and various meta-analyzes have highlighted the clinical validity [54]. The practice has demonstrated the relative ease of administration and the considerable degree of safety. In specific way, the SLIT has a mechanism that induce the tolerance to specific allergen by $\mathrm{T}$ cells reducing cytokine Th2 and stimulating block antibody like IgA, IgG1 e IgG4 [55]. For different property the SLIT to other way of administration, like subcutaneous route, is preferred because has greater safety and efficacy, even if the effect seem to be delayed. For this reason authors would be use this technique to immunotherapy treatment in phase of maintenance, also because prevent chronic infectious disease and of allergens as in SLIT is independent from swallowing the allergen and from any contact of the allergen with the mucosa of the lower gastrointestinal tract. In conclusions SLIT is considered a valid way to the sublingual administration route that might induce immunological tolerance toward allergens involveing cells and mediators specific of oral and intestinal mucosa.

\section{REFERENCES}

[1] Tattersfield, A.E., Knox, A.J., Britton, J.R. and Hall, I.P. (2002) Asthma. Lancet, 360, 1313-1322. doi:10.1016/S0140-6736(02)11312-2

[2] Kabesch, M., Schedel, M., Carr, D., Woitsch, B., Fritzsch, C., Weiland, S.K. and von Mutius, E. (2006) IL-4/IL-13 pathway genetics strongly influence serum IgE levels and childhood asthma. Journal of Allergy and Clinical Immunology, 117, 269-274. doi:10.1016/j.jaci.2005.10.024

[3] Rigoli, L., Briuglia, S., Caimmi, S., Ferrau, V., Gallizzi, R., Leonardi, S., et al. (2011) Gene-environment interactions in childhood asthma. International Journal of Immunopathology and Pharmacology, 24, 41-47.

[4] Pascual, R.M. and Peters, S.P. (2005) Airway remodeling contributes to the progressive loss of lung function in asthma: An overview. Journal of Allergy and Clinical Immunology, 116, 477-486. doi:10.1016/j.jaci.2005.07.011

[5] Pepe, C., Foley, S., Shannon, J., Lemiere, C., Olivenstein, R., Ernst, P., et al. (2005) Differences in airway remodeling between subjects with severe and moderate asthma. Journal of Allergy and Clinical Immunology, 116, 544549. doi:10.1016/j.jaci.2005.06.011

[6] Tang, M.L., Wilson, J.W., Stewart, A.G. and Royce, S.G. (2006) Airway remodelling in asthma: Current understanding and implications for future therapies. Pharmacology \& Therapeutics, 112, 474-488. doi:10.1016/j.pharmthera.2006.05.001

[7] Miraglia del Giudice, M., Marseglia, G.L., Leonardi, S., Tosca, M.A., Marseglia, A., Perrone, L., et al. (2011) Fractional exhaled nitric oxide measurements in rhinitis and asthma in children. International Journal of Immunopathology and Pharmacology, 24, 29-32.

[8] Takeda, N., Sumi, Y., Prefontaine, D., Al Abri, J., Al Heialy, N., Al-Ramli, W., et al. (2009) Epithelium-derived chemokines induce airway smooth muscle cell migration. Clinical \& Experimental Allergy, 39, 1018-1026. doi:10.1111/j.1365-2222.2009.03238.x

[9] Holgate, S.T. (2008) The airway epithelium is central to the pathogenesis of asthma. Allergology International, 57, 1-10. doi:10.2332/allergolint.R-07-154

[10] De Boer, W.I., Sharma, H.S., Baelemans, S.M., Hoogsteden, H.C., Lambrecht, B.N. and Braunstahl, G.J. (2008) Altered expression of epithelial junctional proteins in atopic asthma: Possible role in inflammation. Canadian Journal of Physiology and Pharmacology, 86, 105-112. doi:10.1139/Y08-004

[11] Davies, D.E. (2000) The role of the epithelium in airway remodelling in asthma. Proceedings of the American Thoracic Society, 96, 678-682.

[12] Dekkers, B.G., Maarsingh, H., Meurs, H. and Gosens, R. (2009) Airway structural components drive airway smooth muscle remodelling in asthma. Proceedings of the American Thoracic Society, 6, 683-692. doi:10.1513/pats.200907-056DP

[13] James, A.L. and Wenzel, S. (2007) Clinical relevance of airway remodelling in airway diseases. European Respiratory Journal, 30, 134-155. doi:10.1183/09031936.00146905

[14] Hackett, T.L., Shaheen, F., Johnson, A., Wadsworth, S., Pechkovsky, D.V., Jacoby, D.B., et al. (2008) Characterization of side population cells from human airway epithelium. Stem Cells, 26, 2576-2585. doi:10.1634/stemcells.2008-0171

[15] Boxall, C., Holgate, S.T. and Davies, D.E. (2006) The contribution of transforming growth factor-\{beta $\}$ and epidermal growth factor signalling to airway remodelling in chronic asthma. European Respiratory Journal, 27, 208-229. doi:10.1183/09031936.06.00130004

[16] Koppelman, G.H., Meyers, D.A., Howard, T.D., Zheng, S.L., Hawkins, G.A., Ampleford, E.J., et al. (2009) Identification of PCDH1 as a novel susceptibility gene for 
bronchial hyper-responsiveness. American Journal of Respiratory and Critical Care Medicine, 180, 929-935. doi:10.1164/rccm.200810-16210C

[17] Barbato, A., Turato, G., Baraldo, S., Bazzan, E., Calabrese, F., Panizzolo, C., et al. (2006) Epithelial damage and angiogenesis in the airways of children with asthma. American Journal of Respiratory and Critical Care Medicine, 174, 975-981. doi:10.1164/rccm.200602-1890C

[18] Jeffery, P.K. (2001) Remodeling in asthma and chronic obstructive lung disease. American Journal of Respiratory and Critical Care Medicine, 164, S28-S38.

[19] Berger, P., Girodet, P.O., Begueret, H., Ousova, O., Perng, D.W., Marthan, R., et al. (2003) Tryptase-stimulated human airway smooth muscle cells induce cytokine synthesis and mast cell chemotaxis. The FASEB Journal, 17, 2139-2141.

[20] Leonardi, S., Vitaliti, G., Marseglia, G.L., Caimmi, D., Lionetti, E., Miraglia del Giudice, M., et al. (2012) Function of the airway epithelium in asthma. Journal of Biological Regulators \& Homeostatic Agents, 26, 41-48.

[21] Ramos-Barbon, D., Fraga-Iriso, R., Brienza, N.S., MonteroMartinez, C., Verea-Hernando, H., Olivenstein, R., et al. (2010) T cells localize with proliferating smooth muscle alphaactin+ cell compartments in asthma. American Journal of Respiratory and Critical Care Medicine, 182, 317324. doi:10.1164/rccm.200905-0745OC

[22] Holgate, S.T. (2007) Epithelium dysfunction in asthma. Journal of Allergy and Clinical Immunology, 120, 12331244. doi:10.1016/j.jaci.2007.10.025

[23] Cohen, L., Tarsi, J., Ramkumar, T., Horiuchi, T.K., Cochran, R., et al., and the NHLBI Severe Asthma Research Program (SARP) (2007) Epithelial cell proliferation contributes to airway remodeling in severe asthma. American Journal of Respiratory and Critical Care Medicine, 176, 138-145.

[24] Xiao, C., Puddicombe, S.M., Field, S. and Davies, D.E. (2011) Detective epithelial barrier function in asthma. Journal of Allergy and Clinical Immunology, 128, 549556. doi:10.1016/j.jaci.2011.05.038

[25] Hammad, H. and Lambrecht, B.N. (2008) Dendritic cells and epithelial cells: Linking innate and adaptive immunity in asthma. Nature Reviews Immunology, 8, 193-204. doi:10.1038/nri2275

[26] Ziegler, S.F. and Liu, Y.J. (2007) Thymic stromal lymphopoietin in normal and pathogenic $\mathrm{T}$ cell development and function. Nature Immunology, 7, 709-714. doi:10.1038/ni1360

[27] Allakhverdi, Z., Comeau, M.R., Jessup, H.K., Yoon, B.R., Brewer, A., Chartier, S., et al. (2007) Thymic stromal lymphopoietin is released by human epithelial cells in response to microbes, trauma, or inflammation and potently activates mast cells. The Journal of Experimental Medicine, 204, 253-258. doi:10.1084/jem.20062211

[28] Ying, S., O’Connor, B., Ratoff, J., Meng, Q., Mallett, K., Cousins, D., et al. (2005) Thymic stromal lymphopoietin expression is increased in asthmatic airways and correlates with expression of Th2-attracting chemokines and disease severity. The Journal of Immunology, 174, 81838190.

[29] Pégorier, S., Arouche, N., Dombret, M.C., Aubier, M. and Pretolani, M. (2007) Augmented epithelial endothelin-1 expression in refractory asthma. Journal of Allergy and Clinical Immunology, 120, 1301-1307. doi:10.1016/j.jaci.2007.09.023

[30] Zhao, Y., Usatyuk, P.V., Gorshkova, I.A., He, D., Wang, T., Moreno-Vinasco, L., et al. (2009) Regulation of COX2 expression and IL-6 release by particulate matter in airway epithelial cells. American Journal of Respiratory Cell and Molecular Biology, 40, 19-30. doi:10.1165/rcmb.2008-01050C

[31] Gereke, M., Jung, S., Buer, J. and Bruder, D. (2009) Alveolar type II epithelial cells present antigen to CD4(1) T cells and induce Foxp3(1) regulatory T cells. American Journal of Respiratory and Critical Care Medicine, 179, 344-355. doi:10.1164/rccm.200804-592OC

[32] Schmidt, M., Sun, G., Stacey, M.A., Mori, L. and Mattoli, S. (2003) Identification of circulating fibrocytes as precursors of bronchial myofibroblasts in asthma. The Journal of Immunology, 171, 380-389.

[33] Del Giudice, M.M., Pedullà, M., Brunese, F.P., Capristo, A.F., Capristo, C., Tosca, M.A., Leonardi, S. and Ciprandi, G. (2010) Neutrophilic cells in sputum of allergic asthmatic children. European Journal of inflammation, 8, 151-156.

[34] O’Byrne, P.M., Pedersen, S., Lamm, C.J., Tan, W.C., Busse, W.W.; START Investigators Group (2009) Severe exacerbations and decline in lung function in asthma. American Journal of Respiratory and Critical Care Medicine, 179, 19-24. doi:10.1164/rccm.200807-1126OC

[35] Turato, G., Barbato, A., Baraldo, S., Zanin, M.E., Bazzan, E., Lokar-Oliani, K., et al. (2008) Nonatopic children with multitrigger wheezing have airway pathology comparable to atopic asthma. American Journal of Respiratory and Critical Care Medicine, 178, 476-482. doi:10.1164/rccm.200712-18180C

[36] Saunders, R., Siddiqui, S., Kaur, D., Doe, C., Sutcliffe, A., Hollins, F., et al. (2009) Fibrocyte localization to the airway smooth muscle is a feature of asthma. Journal of Allergy and Clinical Immunology, 123, 376-384. doi:10.1016/j.jaci.2008.10.048

[37] El-Shazly, A., Berger, P., Girodet, P.O., Ousova, O., Fayon, M., Vernejoux, J.M., et al. (2006) Fraktalkine produced by airway smooth muscle cells contributes to mast cell recruitment in asthma. The Journal of Immunology, 176, 1860-1868.

[38] Hollins, F., Kaur, D., Yang, W., Cruse, G., Saunders, R., Sutcliffe, A., et al. (2008) Human airway smooth muscle promotes human lung mast cell survival, proliferation, and constitutive activation: Cooperative roles for CADM1, stem cell factor, and IL-6. The Journal of Immunology, 181, 2772-2780.

[39] Koff, J.L., Shao, M.X., Ueki, I.F. and Nadel, J.A. (2008) Multiple TLRs activate EGFR via a signaling cascade to produce innate immune responses in airway epithelium. American Journal of Physiology-Lung Cellular and Molecular Physiology, 294, L1068-L1075. 
doi:10.1152/ajplung.00025.2008

[40] Kunzmann, S., Schmidt-Weber, C., Zingg, J.M., Azzi, A., Kramer, B.W., Blaser, K., et al. (2007) Connective tissue growth factor expression is regulated by histamine in lung fibroblasts: Potential role of histamine in airway remodelling. Journal of Allergy and Clinical Immunology, 119, 1398-1407. doi:10.1016/j.jaci.2007.02.018

[41] Léguillette, R., Laviolette, M., Bergeron, C., Zitouni, N., Kogut, P., Solway, J., et al. (2009) Myosin, transgelin, and myosin light chain kinase: Expression and function in asthma. American Journal of Respiratory and Critical Care Medicine, 179, 194-204. doi:10.1164/rccm.200609-13670C

[42] Simcock, D.E., Kanabar, V., Clarke, G.W., Mahn, K., Karner, C., O’Connor, B.J., et al. (2008) Induction of angiogenesis by airway smooth muscle from patients with asthma. American Journal of Respiratory and Critical Care Medicine, 178, 460-468. doi:10.1164/rccm.200707-10460C

[43] Chetta, A., Zanini, A., Foresi, A., D’Ippolito, R., Tipa, A., Castagnaro, A., et al. (2005) Vascular endothelial growth factor up-regulation and bronchial wall remodelling in asthma. Clinical \& Experimental Allergy, 35, 1437-1442. doi:10.1111/j.1365-2222.2005.02360.X

[44] Lommatzsch, M., Lindner, Y., Edner, A., Bratke, K., Kuepper, M. and Virchow, J.C. (2009) Adverse effects of salmeterol in asthma: A neuronal perspective. Thorax, 64, 763-769. doi:10.1136/thx.2008.110916

[45] Araya, J., Cambier, S., Markovics, J.A., Wolters, P., Jablons, D., Hill, A., et al. (2007) Squamous metaplasia amplifies pathologic epithelial-mesenchymal interactions in COPD patients. Journal of Clinical Investigation, 117, 3551-3562. doi:10.1172/JCI32526

[46] Rock, J.R., Randell, S.H. and Hogan, B.L.M. (2010) Airway basal steam cells: A perspective in their roles in epithelial homeostasis and remodelling. Disease Models and Mechanisms, 3, 545-556. doi:10.1242/dmm.006031

[47] Macchiarini, P., Jungebluth, P., Go, T., Asnaghi, M.A., Rees, L.E., Cogan, T.A., et al. (2008) Clinical transplanttation of a tissue-engineered airway. Lancet, 372, 20232030. doi:10.1016/S0140-6736(08)61598-6
[48] Bousquet, J., Schünemann, H.J., Samolinski, B., Demoly, P., Baena-Cagnani, C.E., et al. (2012) Allergic Rhinitis and its Impact on Asthma (ARIA): Achievements in 10 years and future needs. Journal of Allergy and Clinical Immunology, 130, 1049-1062.

[49] La Rosa, M., Lionetti, E., Leonardi, S., Salpietro, A., Bianchi, L., Salpietro, C., et al. (2011) Specific immunotherapy in children: The evidence. International Journal of Immunopathology and Pharmacology, 24, 69-78.

[50] Vitaliti, G., Leonardi, S., Miraglia del Giudice, M., Salpietro, A., Artusio, L., Caimmi, D., Arrigo, T., Salpietro, C., Ciprandi, G. and La Rosa, M. (2012) Mucosal immunity and sublingual immunotherapy in respiratory disorders. Journal of Biological Regulators \& Homeostatic Agents, 26, 85-94.

[51] Bousquet, J., Scheinmann, P., Guinnepain, M.T., PerrinFayolle, M., Sauvaget, J., Tonnel, A.B., et al. (1999) Sublingual-swallow immunotherapy (SLIT) in patients with asthma due to housedust mites: A double-blind, placebo-controlled study. Allergy, 54, 249-260. doi:10.1034/j.1398-9995.1999.00916.x

[52] La Rosa, M., Ranno, C., André, C., Carat, F., Tosca, M.A. and Canonica, G.W. (1999) Double-blind placebo-controlled evaluation of sublingual swallow immunotherapy with standardized Parietaria judaica extract in children with allergic rhinoconjunctivitis. Journal of Allergy and Clinical Immunology, 104, 425-432. doi:10.1016/S0091-6749(99)70388-X

[53] Miraglia del Giudice, M., Marseglia, A., Leonardi, S., La Rosa, M., Salpietro, C., Arrigo, T., et al. (2011) Allergic rhinitis and quality of life in children. International Journal of Immunopathology and Pharmacology, 24, 25-28.

[54] Bush, R.K., Swenson, C., Falherg, B., Evans, M.D., Esch, R. and Busse, W.W. (2011) House dust mite sublingual immunotherapy: Results of a US trial. Journal of Allergy and Clinical Immunology, 127, 974-981. doi:10.1016/j.jaci.2010.11.045

[55] Keles, S, Karakoc-Aydiner, E., Ozen, A., et al. (2011) A novel approach in allergeb specific immunotherapy: Combination of sublingual and subcutaneous routes. Journal of Allergy and Clinical Immunology, 128, 808-815. doi:10.1016/j.jaci.2011.04.033 


\section{EVIDENCES}

*Asthma is a chronic, multisystem, multicellular disease of airway frequently characterized by different exacerbations of inflammatory.

${ }^{*}$ Asthma exacerbations are associated with an increase of airway inflammation, probabily caused by respiratory infections, exposure to allergens, or an impaired repair mechanism with inability to restore cell-cell contacts upon damage, as frequently in susceptible individuals.

"Chronic inflammation of the airways is always associated with the damage and the repair of the bronchial epithelium, which causes structural and functional changes known as remodeling.

*Epithelial cells possess microbial detection mechanisms. This cell-type-specific innate immune recognition equipment enables them to respond to antigens and allergens, thereby initiating the first step in the hostpathogen interaction.

*The airway epithelium is known to be a major source of pro-inflammatory mediators. Basal cells (BCs) are a common feature of stratified epithelia that drives both homeostasis of the normal epithelium and probably contribute to disease susceptibility, initiation and progresssion.

*The knowledge of the pathological role of the airway epithelium in developing asthma could be useful to physicians to develop target drugs in order to better treatment and the management of bronchial hyper-reactivity.

*The role of specific immunotherapy (SIT) to overcome the problem of the evolution of asthma. 